\title{
Shrinking the Variance-Covariance Matrix: Simpler is Better
}

\section{Muhammad Husnain, ${ }^{*}$ Arshad Hassan, ${ }^{* *}$ and Eric Lamarque ${ }^{* * *}$}

\begin{abstract}
This study focuses on the estimation of the covariance matrix as an input to portfolio optimization. We compare 12 covariance estimators across four categories - conventional methods, factor models, portfolios of estimators and the shrinkage approach - applied to five emerging Asian economies (India, Indonesia, Pakistan, the Philippines and Thailand). We find that, in terms of the root mean square error and risk profile of minimum variance portfolios, investors gain no additional benefit from using the more complex shrinkage covariance estimators over the simpler, equally weighted portfolio of estimators in the sample countries.
\end{abstract}

Keywords: Variance-covariance matrix, mean-variance criteria, portfolio management.

JEL classification: C13, C51, C52, G11, G15.

\section{Introduction}

The concept of mean-variance optimization was introduced by Markowitz (1952). Although studies such as Jagannathan and Ma (2003) and Chan, Karceski and Lakonishok (1999) support the use of the standard mean-variance framework for optimal portfolio construction, it has been criticized on a number of fronts. Michaud (1989) terms the concept an "enigma" while Disatnik and Benninga (2007) argue that it yields questionable results. There are two main approaches to dealing with the problems presented by traditional mean-variance optimization. The theoretical approach focuses on the assumptions and notional aspects of the mean-variance framework, while the implementation approach looks at how investors can estimate the expected return vector and covariance matrix of asset classes in order to use the framework successfully.

\footnotetext{
* Department of Management Sciences, Capital University of Science and Technology, Islamabad; research fellow, IAE de Paris, Université Paris 1 Panthéon-Sorbonne.

** Associate Professor, Faculty of Management and Social Sciences, Capital University of Science and Technology, Islamabad.

**** Professor, IAE de Paris, Université Paris 1 Panthéon-Sorbonne.
} 
This study examines the literature on the implementation approach to mean-variance optimization and the estimation of the covariance matrix, which is seen as the most troubling aspect of the framework (Ledoit \& Wolf, 2003). Elton and Gruber (1973) and DeMiguel, Garlappi and Uppal (2009) reinforce the importance of the covariance estimator in implementing mean-variance optimization successfully.

The sample covariance matrix is the conventional measure for estimating the pair-wise covariances of asset classes, based primarily on past covariances. Pafka and Kondor (2004), Michaud (1989), and Jobson and Korkie (1980) have criticized this method of estimating pair-wise covariances. Specifically, it is prone to errors when the number of underlying asset classes is larger than the sample. Michaud (1989) labels this phenomenon "error maximization." Sharpe (1963) proposes a relatively intuitive way of explaining covariances through a common factor - the market factor. Blume (1971), Vasicek (1973) and King (1966) try to improve the estimator by considering the mean-reverting tendency of betas, adjusting their variation and taking into account other factors beyond the single common factor, respectively.

Statistical and nontheory-based measures, such as principal component analysis (PCA), can also be used to identify factors relating to historical sample covariances. Elton and Gruber (1973) suggest using average correlation-based covariance estimators. While the literature on covariance estimators is too extensive to survey here, we agree that the standard method of estimating the covariance matrix is prone to either estimation or specification errors. Given the errors and numerical instability of estimators, DeMiguel et al. (2009) conclude empirically that nontheory-based diversification outperforms the more sophisticated asset allocation strategies.

The financial literature applies a fundamental principle of statistics to optimize between the estimation error and specification error. Bengtsson and Holst (2002), Chan et al. (1999), Jagannathan and Ma (2003), Ledoit and Wolf $(2003,2004)$ and Wolf (2004) show empirically that shrinkage estimators and a portfolio of estimators are best suited to covariance estimation. As per the decision theory in statistics, there is an optimal point between the specification error and estimation error. According to Stein (1956), this optimal point can be determined by a weighted average of both estimators.

Ledoit and Wolf (2003) suggest using the Bayesian shrinkage approach to optimization in relation to the single-index covariance 
estimator and sample covariance estimator. This process guarantees to reduce the estimation error in the sample covariance without producing much of a specification error. It results in a shrinkage matrix whereby all the covariances (off-diagonal elements) of the conventional sample matrix are shrunk without changing the diagonal elements. Ledoit and Wolf (2004) shrink the sample covariance toward a constant correlation covariance estimator. Jagannathan and Ma (2003) conceptualize a portfolio of covariance estimators, which challenges the more complex Ledoit and Wolf (2003) estimator, and involves using the equally weighted average of the sample covariance estimator and any other covariance estimator.

Both the shrinkage estimator and equally weighted estimator are supposed to be better than the sample covariance estimator. Of these approaches, shrinkage estimators are theoretically more complex than the simpler, equally weighted average of a portfolio of estimators. Disatnik and Benninga (2007) use data from the New York Stock Exchange to confirm that investors have no additional benefit to gain from using shrinkage estimators over an equally weighted portfolio of covariance estimators. However, the literature on covariance estimation offers no real consensus on the relative merits of sophisticated versus simple estimators in the context of equity markets in emerging Asian economies.

With this in mind, we compare 12 covariance estimators across four groups - conventional methods, factor models, a portfolio of estimators and the shrinkage approach - applied to five emerging Asian economies (India, Indonesia, Pakistan, the Philippines and Thailand). For this purpose, we use the equity classification from the Global Industry Classification Standard (GICS) developed by Morgan Stanley Capital International and Standard \& Poor's. We use two different criteria to assess covariance estimators: the root mean square error (RMSE) and the risk associated with the minimum variance portfolio (MVP). The RMSE of a pair-wise covariance matrix focuses on accuracy, while the MVP risk measure gauges the effectiveness of estimators in selecting an MVP. We find that the sample covariance matrix remains a poor estimator in terms of the RMSE and MVP, while the equally weighted average of covariance estimators performs better than the shrinkage estimators proposed by Ledoit and Wolf $(2003,2004)$.

Section 2 describes the dataset and research methodology used, including our criteria for comparison. Section 3 presents the study's empirical findings, followed by a discussion of the results in Section 4 . Section 5 concludes the paper. 


\section{Dataset and Research Methodology}

We have used the Bloomberg database to collect data on the sample countries. The sample period spans 22 March 2002 to 30 October 2015 on a biweekly basis. This is divided into two subsamples: from 22 March 2002 to 2 January 2009 and from 16 January 2009 to 30 October 2015. The covariance matrices are estimated based on the first subsample. The second window is used to provide the ex-post accuracy of the covariance matrix.

This study develops equally weighted indices based on the GICS, which consists of ten sectors for each sample country: the consumer discretionary sector, consumer staples, energy, financials, healthcare, industrials, information technology, materials, telecommunication services and utilities. Table 1 gives details of the selected representative equity indices for the stock market of each country.

Table 1: Summary of selected equity indices for sample countries

\begin{tabular}{lll}
\hline Country & \multicolumn{1}{c}{ Stock market } & \multicolumn{1}{c}{ Representative index } \\
\hline India & Bombay Stock Exchange & S\&P BSE Sensex (cap-weighted) \\
Indonesia & Indonesian Bursa Efek Jakarta & JCI index (mod cap-weighted) \\
Pakistan & Karachi Stock Exchange & KSE-100 index (cap-weighted) \\
Philippines & Philippine Stock Exchange & PSEi index (cap-weighted) \\
Thailand & Stock Exchange of Thailand & SET index (cap-weighted) \\
\hline
\end{tabular}

The continuous compounded return $\left(R_{i, t}\right)$ on each asset class is calculated by the formula $R_{i, t}=\ln \left(P_{t} / P_{t-1}\right)$. Here, $P_{t}$ and $P_{t-1}$ are the returns in the current and previous period for the asset class, respectively.

\subsection{Estimation of Variance-Covariance Matrix}

The variance-covariance matrix is a square matrix of the variances and covariances of the asset classes concerned. It contains the variances of each asset class as diagonal entries, while the off-diagonal entries comprise the covariances of all possible pairs of the asset classes. Simply put, the variance is the squared mean deviation while the covariance indicates how two asset classes change together. Mathematically, a variance-covariance matrix can be written as follows: 


$$
\Sigma=\left[\begin{array}{cccc}
\sum x_{1}^{2} / n & \sum x_{1} x_{2} / n & \cdots & \sum x_{1} x_{i} / n \\
\sum x_{2} x_{1} / n & \sum x_{2}^{2} / n & \ldots & \sum x_{2} x_{i} / n \\
\cdots & \cdots & \cdots & \cdots \\
\sum x_{i} x_{1} / n & \sum x_{i} x_{2} / n & \cdots & \sum x_{i}^{2} / n
\end{array}\right]
$$

Here, $\Sigma$ is the variance-covariance matrix $(i * i), n$ is the number of data points in each asset class, $x_{i}$ represents the mean deviation, $\sum x_{1}^{2} / n$ is the variance of the $i$ th asset class, and $\sum x_{i} x_{j} / n$ is the covariance between asset classes $i$ and $j$. This study compares the performance of 12 covariance matrices across conventional methods, factor models, a portfolio of estimators and the shrinkage approach. The matrices are outlined below.

\subsubsection{Sample Covariance Matrix}

For any vector $l \in R^{n}$, where the sample variance is $\sigma^{2}$ and the sample average is $\hat{x}$, then:

$$
\hat{l}=\frac{1}{n}\left(l_{1}+l_{2}+l_{3}+\cdots+l_{n}\right), \sigma^{2}=\frac{1}{n}\left(\left(l_{1}-\hat{l}\right)^{2}+\left(l_{2}-\hat{l}\right)^{2}+\cdots+\left(l_{n}-\hat{l}\right)^{2}\right)
$$

Let $X=\left[x_{1}+x_{2}+x_{3}+\cdots+x_{n}\right] \in R^{m * n}$ where every column $x_{i}$ represents an observation in $R^{m}$. For the variance, we have the values obtained from projecting the data along a line in the direction $\tau \in R^{m}$ such that:

$$
l=\left(\tau^{T} x_{1}+\tau^{T} x_{2}+\tau^{T} x_{3}+\cdots \tau^{T} x_{n}\right)=\tau^{T} X \in R^{n}
$$

The corresponding sample variance and sample mean are:

$$
\sigma^{2}(\tau)=\frac{1}{n} \sum_{k=1}^{n}\left(\tau^{T} x_{k}-\tau^{T} \hat{x}\right)^{2}, \hat{l}=\tau^{T} \hat{x}
$$

In this equation, the sample mean is $\hat{x}=\frac{1}{n}\left(x_{1}+x_{2}+x_{3}+\cdots+\right.$ $\left.x_{n}\right) \in R^{m}$ and the variance with direction $\tau$ in quadratic form is:

$$
\sigma^{2}(\tau)=\frac{1}{m} \sum_{k=1}^{m}\left[\tau^{T}\left(x_{k}-\hat{x}\right)\right]^{2}=\tau^{T} \Sigma \tau
$$


In the last expression, the sample variance-covariance is represented by $\Sigma$ and can be written as:

$$
\Sigma_{\text {sample }}=\frac{1}{n} \sum_{k=1}^{n}\left(x_{k}-\hat{x}\right)\left(x_{k}-\hat{x}\right)^{T}
$$

The covariance matrix in equation (1) is symmetrical, positive and semi-definite, and can be used to find the variance in any direction.

\subsubsection{Constant Correlation (Overall Mean) Covariance Matrix}

Elton and Gruber (1973) estimate the covariance matrix on the assumption that the variance of the return on each asset class is the sample return and that the covariance is associated by the same coefficient of correlation. For this, we use the average correlation coefficient of all the asset classes in question. Chan et al. (1999) also claim that this covariance matrix is more appropriate than its alternatives. We know that $\sigma_{l m}=$ $\rho_{l, m} \sigma_{l} \sigma_{m}$ and, therefore:

$$
\sigma_{l m}=\left\{\begin{array}{r}
\sigma_{l m}=\sigma_{l}^{2} \text { if } l=m \\
\sigma_{l m}=\rho_{l, m} \sigma_{l} \sigma_{m} \text { if } l \neq m
\end{array}\right.
$$

\subsubsection{Single-Index Covariance Matrix}

Sharpe (1963) presents the single-index formula and assumes that the return on any asset class can be written as a linear combination of the market portfolio. Hence, there is a significant, positive linear relationship between asset returns and market portfolios, which can be expressed as:

$$
Y_{i t}=\alpha_{i}+\gamma_{i} x_{t}+\varepsilon_{t}
$$

where $x_{t}$ denotes the market portfolio, which is uncorrelated with the error term. Further, $E\left(\varepsilon_{i t} \varepsilon_{j t}\right)=0$. The variance $\left(\operatorname{var}\left(\varepsilon_{i t}\right)=\delta_{i i}\right)$ within the asset classes is unchanged. The covariance matrix $\left(\sigma_{i j}\right)$ is expressed as:

$$
\sigma_{i j}=\beta \sigma^{2} \beta^{T}+\varphi
$$

Here, $\beta, \sigma^{2}$ and $\varphi$ denote the vector of the slope, the variance of the market and the matrix of the variance of the error term, respectively. The covariance matrix under the single-index model takes this form:

$$
\Sigma_{\text {single index }}=b \sigma^{2} \hat{b}+\omega
$$


where $b, \sigma^{2}$ and $\omega$ are the vector of slope estimates, the sample variance of the market and the matrix of the variance of error term estimates, respectively.

\subsubsection{Principal Component Model}

PCA is used to examine the underlying motives for co-movement among asset classes. It does so without any economic justification and transforms the vector space of $K$ asset classes into $K$ factors. PCA uses the singular value decomposition of the sample covariance. The $j$ th factor out of $K$ is the linear combination of $K$ asset classes. We also assume that there is no correlation among the factors. Mathematically, asset returns and the sample covariance take the following form:

$$
\begin{aligned}
& R_{j}^{e}=\sum_{j=1}^{k} \tau_{i j} F_{j} \\
& \Sigma=\tau Z_{F} \dot{\tau}
\end{aligned}
$$

Here, $\tau$ is a matrix of eigenvectors of order $1^{*} N$ and $Z$ is a matrix of eigenvalues of order $N^{*} N$. Since the objective of PCA is to cut dimensions, out of $K$ factors we can select only the first $G$ factors if $\sum_{a=0}^{G} \sigma_{F, a}^{2} /$ $\sum_{a=0}^{K} \sigma_{F, a}^{2} \cong 1$. Therefore, the PCA-based covariance matrix of the first $G$ factors is estimated as follows:

$$
\Sigma=\tilde{\tau} \widetilde{Z_{F}} \tilde{\tau}^{\prime}+Z_{\varepsilon}
$$

Here, $\tau$ is a matrix of the first $G$ eigenvectors and $Z$ is a diagonal matrix of the first $G$ eigenvalues. We use equation (4) to estimate the covariance matrix based on PCA.

\subsubsection{Shrinkage Variance-Covariance Matrix}

The single-index covariance matrix and the sample covariance are two sides of the same coin in that the first is a one-factor model while the second is an $N$-factor model. Generally, a true estimator is held to be an $m$ factor model such that $N>m>1$. Ledoit and Wolf (2003) suggest that the single-index covariance has a specification problem, but the sample matrix also has an inherent estimation problem.

Stein (1956) shows that an optimal point can be determined by taking the weighted average of both estimators. This method involves 
shrinking the sample matrix toward the fixed (diagonal) target. Jorion (1986) suggests that this shrinking plays a vital role in portfolio selection. Assuming $\psi$ and $\phi$ are the parameters of unrestricted high-dimension and restricted low-dimension sub-models, respectively, we can obtain the corresponding estimates $L=\hat{\psi}$ and $K=\hat{\phi}$ from the observed data. $L$ has a high variance as it requires more fitted parameters than $K$, but $K$ is theoretically biased. The estimator can be written as:

$$
\Sigma=\theta * K+(1-\theta) * L
$$

Here, $L$ denotes the sample covariance, $K$ is the target matrix (highly structured estimator) and $\theta$ is the weight of $K$ in the convex linear combination between $K$ and $L$. The shrinkage intensity of $\theta$ ranges from 0 to 1 . If the value of $\theta$ is 0 , we return to the sample matrix and it implies no shrinkage. On the other hand, if $\theta=1$, then there is complete shrinkage and the resulting covariance matrix is equal to the target $K$. The question is whether to fix the value of $\theta$ or let it be determined by minimizing the following loss function (mean square error):

$$
R(\theta)=E\left(\sum_{i=1}^{p}\left(l_{i}^{*}-\psi_{i}\right)^{2}\right)
$$

Ledoit and Wolf's (2003) analytical formula for determining the optimal shrinkage intensity $(\theta)$ involves shrinking the sample covariance matrix toward the single-index covariance matrix. This ensures that the mean square error is minimized without any assumption about distribution. Given the first and second moments of $L$ and $K$, the squared error loss function from equation (6) is:

$$
\begin{aligned}
R(\theta) & =\sum_{i=1}^{p} \operatorname{var}\left(l_{i}^{*}\right)+\left[E\left(l_{i}^{*}\right)-\psi_{i}\right]^{2} \\
R(\theta)= & \sum_{i=1}^{p} \operatorname{var}\left(\theta t_{i}+(1-\theta) l_{i}\right)+\left[E\left(\theta t_{i}+(1-\theta) l_{i}\right)-\psi_{i}\right]^{2} \\
R(\theta)= & \sum_{i=1}^{p} \theta^{2} \operatorname{var}\left(t_{i}\right)+(1-\theta)^{2} \operatorname{var}\left(l_{i}\right)+2 \theta(1-\theta) \operatorname{cov}\left(l_{i}, t_{i}\right) \\
& \quad+\left[\theta E\left(t_{i}-l_{i}\right)+\operatorname{bias}\left(l_{i}\right)\right]^{2}
\end{aligned}
$$

Minimizing this function with respect to $\theta$, we have: 


$$
\theta^{*}=\frac{\sum_{i=1}^{p} \operatorname{var}\left(l_{i}\right)-\operatorname{cov}\left(l_{i}, t_{i}\right)-\operatorname{bias}\left(l_{i}\right) E\left(t_{i}-l_{i}\right)}{\sum_{i=1}^{p} E\left[\left(t_{i}-l_{i}\right)^{2}\right]}
$$

If $L$ is an unbiased estimator of $\psi$, then the above expression can be written as:

$$
\theta^{*}=\frac{\sum_{i=1}^{p} \operatorname{var}\left(l_{i}\right)-\operatorname{cov}\left(l_{i}, t_{i}\right)}{\sum_{i=1}^{p} E\left[\left(t_{i}-l_{i}\right)^{2}\right]}
$$

We use expression (7) to compute the optimal shrinkage intensity $(\theta)$ (see Ledoit \& Wolf, 2003). Ledoit and Wolf (2004) shrink the sample covariance matrix toward the constant correlation covariance estimator and propose a formula for computing the optimal shrinking intensity. Bengtsson and Holst (2002) shrink the sample covariance matrix to the $k$ factor principal component model, while Kwan (2011) shows how to shrink the sample covariance toward the diagonal matrix. Consistent with the literature, we use three types of targets: the diagonal target, the singleindex covariance and the constant correlation covariance matrix. For further discussion of these shrinkage estimators, see Ledoit and Wolf (2003, 2004) and Kwan (2011).

\subsubsection{Portfolio of Estimators}

Jagannathan and Ma (2003) criticize the concept of optimally weighted intensity presented by Ledoit and Wolf (2003), and introduce equally weighted covariance estimators instead. In line with Jagannathan and Ma (2003), Liu and Lin (2010), and Disatnik and Benninga (2007), we use the following five equally weighted portfolios of estimators:

- Portfolio of sample matrix and diagonal matrix. In equation (8), $\Sigma_{p 1}$ is the equally weighted average of the sample covariance and diagonal covariance matrix. In the diagonal matrix, all the off-diagonal elements are equal to 0 while the variances of the asset classes are diagonal entries:

$$
\Sigma_{p 1}=\frac{1}{2} \Sigma_{\text {sample }}+\frac{1}{2} \Sigma_{\text {diagonal }}
$$

- Portfolio of sample matrix and single-index matrix. In equation (9), $\Sigma_{p 2}$ is the equally weighted average of the sample covariance and singleindex covariance matrix: 


$$
\Sigma_{p 2}=\frac{1}{2} \Sigma_{\text {sample }}+\frac{1}{2} \sum_{\text {single index }}
$$

- Portfolio of sample matrix and constant correlation covariance matrix. In equation (10), $\Sigma_{p 3}$ is the equally weighted average of the sample covariance and constant correlation (overall mean) covariance matrix:

$$
\Sigma_{p 3}=\frac{1}{2} \Sigma_{\text {sample }}+\frac{1}{2} \Sigma_{\text {overall mean }}
$$

- Portfolio of sample matrix, single-index and constant correlation matrix. In equation (11), $\Sigma_{p 4}$ is the equally weighted average of the sample covariance, the single-index covariance matrix and the constant correlation (overall mean) covariance matrix:

$$
\Sigma_{p 4}=\frac{1}{3} \Sigma_{\text {sample }}+\frac{1}{3} \Sigma_{\text {single index }}+\frac{1}{3} \Sigma_{\text {overall mean }}
$$

- Portfolio of sample matrix, single-index matrix, overall mean matrix and diagonal matrix. In equation (12), $\Sigma_{p 5}$ is the equally weighted average of the sample covariance, the single-index covariance matrix, constant correlation (overall mean) covariance matrix and diagonal matrix:

$$
\Sigma_{p 5}=\frac{1}{4} \Sigma_{\text {sample }}+\frac{1}{4} \Sigma_{\text {single index }}+\frac{1}{4} \Sigma_{\text {overall mean }}+\frac{1}{4} \Sigma_{\text {diagonal }}
$$

\subsubsection{Summary of Covariance Estimators}

We have shown that the structure of alternative covariance matrices can include conventional methods, factor models, a portfolio of estimators and the shrinkage approach (Table 2). The sample matrix is based on historical covariances, but has a lower structure than other covariance estimators. Elton and Gruber (1973) recommend using the historical degree of association to estimate covariance estimators. Similarly, Sharpe (1963) uses systemic risk factors to determine the covariance matrix, although this is criticized on the grounds that it relies on a single systematic risk factor. Arguably, the single-index covariance matrix is more appropriate than the sample covariance on the basis of estimation errors, but it can lead to specification errors. 
Table 2: Summary of variance-covariance methods

\begin{tabular}{|c|c|c|}
\hline Category & & Variance-covariance matrices \\
\hline \multirow{3}{*}{$\begin{array}{l}\text { Conventional } \\
\text { methods }\end{array}$} & $\mathrm{D}$ & Diagonal method \\
\hline & $\mathrm{S}$ & Sample matrix \\
\hline & $\mathrm{CC}$ & Constant correlation model \\
\hline \multirow[t]{2}{*}{ Factor models } & SI & Single-index matrix \\
\hline & PCA & Principal component analysis-based model \\
\hline \multirow{5}{*}{$\begin{array}{l}\text { Portfolio of } \\
\text { estimators }\end{array}$} & P1 & Portfolio of sample matrix and diagonal matrix \\
\hline & P2 & Portfolio of sample matrix and single-index matrix \\
\hline & P3 & Portfolio of sample matrix and constant correlation matrix \\
\hline & P4 & $\begin{array}{l}\text { Portfolio of sample matrix, single-index matrix and constant } \\
\text { correlation matrix }\end{array}$ \\
\hline & P5 & $\begin{array}{l}\text { Portfolio of sample matrix, single-index matrix, constant } \\
\text { correlation matrix and diagonal }\end{array}$ \\
\hline \multirow{3}{*}{$\begin{array}{l}\text { Shrinkage } \\
\text { approaches }\end{array}$} & P6 & Shrinkage to diagonal matrix \\
\hline & P7 & Shrinkage to single-index model \\
\hline & P8 & Shrinkage to constant correlation model \\
\hline
\end{tabular}

Ledoit and Wolf $(2003,2004)$ use an optimal combination of two covariance matrices to yield one covariance estimator by shrinking the sample covariances to the target matrix. Jagannathan and Ma (2003) challenge this approach and propose a simpler, equally weighted average of two or more covariance estimators. Ledoit and Wolf's (2003, 2004) method is theoretically more rigorous, but its empirical results are questionable (Disatnik \& Benninga, 2007). Table 2 summarizes the alternative covariance matrices along with the abbreviations used hereon. It also includes the diagonal method of estimating covariances, which is the basis for other covariance estimators under the categories of "portfolio of estimators" and "shrinkage approaches" (P1, P5, P6).

\subsection{Evaluation of Covariance Estimators}

As mentioned earlier, we use two different assessment criteria to compare the 12 covariance estimators: the RMSE and portfolio allocation. Most of the literature supports the use of these criteria: Liu and Lin (2010) use the RMSE to evaluate the performance of covariance estimators, while Chan et al. (1999), Jagannathan and Ma (2003), and Kyj, Ostdiek and Ensor (2010) use MVP output to gauge alternative covariance estimators.

We use the RMSE to compare the pair-wise accuracy of estimators, initially estimating covariance matrices based on the first subsample window (22 March 2002 to 2 January 2009). The second subsample window 
(16 January 2009 to 30 October 2015) is used to determine the ex-post accuracy of the covariance matrix. This means looking at the difference between the covariance estimators obtained in the two subsample windows. The RMSE is calculated as follows:

$$
R M S E=\sqrt{\frac{M(M-1)}{2} \sum_{j=1}^{M} \sum_{k=1, j \neq k}^{M}\left(\hat{\sigma}_{j k}-\sigma_{j k}\right)^{2}}
$$

Here, $\frac{M(M-1)}{2}$ represents the total pair-wise covariance estimators against the $M^{*} M$ covariance matrix, while $\sigma_{j k}$ and $\hat{\sigma}_{j k}$ are the actual and estimated covariances among $j$ and $k$, respectively. The RMSE is easy to interpret because it has no unit problem and a smaller value is better than a higher one.

Next, we use the MVP method to compare the performance of covariance estimators. An MVP is the only portfolio on the efficient frontier that depends on the covariance matrix, but not on the choice of the vector of returns on asset classes. Although Jagannathan and Ma (2003) argue that a constrained MVP provides better estimates, we use an unrestricted MVP for three reasons. First, our main focus is not the resulting performance of optimal portfolios, but the estimation error that arises when estimating the covariance matrix. Thus, to achieve the maximum estimation error, we use an unconstrained MVP. Second, a constrained MVP is preferable when investors rebalance their portfolios after every period in the out-of-sample window (Chan et al., 1999). However, we focus not on the efficiency of the resultant portfolios, but on estimating the covariance matrix, which is why we use a buy-and-hold MVP. Third, we are interested in the consistency of the RMSE and MVP risk profile as our assessment criteria. Accordingly, we employ an unrestricted MVP in line with Liu and Lin (2010).

The first step is to compute the weights using the MVP under an alternative covariance matrix for the first subsample period. Based on these weights, we note down the return on the MVP in the out-of-sample window (the second subsample) and then calculate the mean risk and mean return for this series. While calculating the RMSE of the pair-wise covariance matrix gauges accuracy, the MVP is a way of looking at the effectiveness of estimators in selecting an MVP. The weight of an MVP of $n$ risky assets is given by:

$$
\operatorname{Min}_{w} w^{T} \sum w \quad \text { s.t. } w^{T} e=1
$$

Using the Lagrangian multiplier $\lambda$, the problem is restated as: 
$\operatorname{Min}_{w} \delta=w^{T} \Sigma w-2 \lambda\left(w^{T} e-1\right)$

The $n$ first-order condition is:

$\frac{\partial \delta}{\partial w}=2 \Sigma w-2 \lambda e=0$

Solving this expression for the weight $w$, we obtain:

$w=\lambda \Sigma^{-1} e$

Let $z$ be a $p \times 1$ column vector defined as $z=\frac{1}{\lambda} w$, which we can write as $z=\Sigma^{-1} e$. Since the sum of the total weights equals $1, z^{T} e=$ $\frac{1}{\lambda} w^{T} e=\frac{1}{\lambda}$. Therefore, the investment weight vector for the MVP is: ${ }^{1}$

$$
w_{m v p}=\frac{z}{z^{T} e}
$$

\section{Empirical Findings}

Table 3 gives the RMSE results for the 12 covariance estimators, indicating the pair-wise covariance estimation and corresponding out-ofsample values. A covariance estimator outperforms other estimators if it has a relatively low RMSE value. From the table, it is evident that the PCAbased covariance estimator consistently outperforms the others, but estimates the covariance matrix without any economic rationale. The sample covariance estimator proves to be a poor estimator of covariance, especially for the Philippines, Pakistan, Indonesia and India. Generally, the overall mean method yields a competitive RMSE using a single-index covariance estimator. By and large, the single-index covariance estimator outperforms the overall mean method for all five countries.

\footnotetext{
${ }^{1}$ The weight of the MVP for $N$ asset classes can also be computed by minimizing the Lagrange function $C$ for portfolio variance:

$$
\begin{aligned}
& \quad \operatorname{Min} \operatorname{var}\left(R_{p}\right)=\sum_{i=1}^{N} \sum_{j=i+1}^{N} w_{i} w_{j} \sigma_{i j} \text { subject to } \sum_{j=1}^{N} w_{j}=1 \\
& C=\sum_{i=1}^{N} \sum_{j=i+1}^{N} w_{i} w_{j} \sigma_{i j}+\lambda_{1}\left(1-\sum_{i=1}^{N} w_{i}\right)
\end{aligned}
$$

Here $w_{i}, w_{j}, \sigma_{i j}$ and $\lambda_{1}$ are the weights, covariance and Lagrange multiplier, respectively.
} 
Table 3: Summary of RMSE under covariance estimators

\begin{tabular}{lcccccc}
\hline Group & Covariance & India & Indonesia & Pakistan & Philippines & Thailand \\
\hline Conventional & S & 0.0293 & 0.0592 & 0.0218 & 0.0461 & 0.0287 \\
methods & CC & 0.0254 & 0.0593 & 0.0170 & 0.0357 & 0.0239 \\
Factor models & SI & 0.0027 & 0.0570 & 0.0169 & 0.0322 & 0.0290 \\
& PCA & 0.0006 & 0.0013 & 0.0003 & 0.0008 & 0.0005 \\
Portfolio of & P1 & 0.0147 & 0.0296 & 0.0109 & 0.0231 & 0.0143 \\
estimators & P2 & 0.0160 & 0.0579 & 0.0175 & 0.0386 & 0.0288 \\
& P3 & 0.0271 & 0.0582 & 0.0156 & 0.0404 & 0.0262 \\
& P4 & 0.0190 & 0.0575 & 0.0148 & 0.0372 & 0.0271 \\
& P5 & 0.0142 & 0.0432 & 0.0111 & 0.0279 & 0.0203 \\
Shrinkage & P6 & 0.0292 & 0.0588 & 0.0217 & 0.0458 & 0.0285 \\
approaches & P7 & 0.0208 & 0.0587 & 0.0176 & 0.0435 & 0.0287 \\
& P8 & 0.0300 & 0.0584 & 0.0129 & 0.0437 & 0.0224 \\
\hline
\end{tabular}

Source: Authors' calculations.

The most important result is the comparison between the complex covariance estimator introduced by Ledoit and Wolf $(2003,2004)$ and the equally weighted portfolio of estimators proposed by Jagannathan and Ma (2003). The results for the shrinkage covariance estimators show that P6 performs worse than P7 and P8 across the sample, barring Thailand. Moreover, P7 has a lower RMSE than P8 for India and the Philippines.

When we compare the RMSE across the equally weighted covariance estimators, then P1 outperforms the other equally weighted estimators for all sample countries except India. P2 and P3 yield comparable RMSE values, but P2 performs better for India, Indonesia and the Philippines. P4 (the equally weighted portfolio of the sample covariance, single-index covariance and constant correlation covariance) outperforms P2 (the equally weighted portfolio of the sample covariance and single-index covariance) and P3 (the equally weighted portfolio of the sample covariance and constant correlation covariance) for Indonesia, Pakistan and the Philippines. For Thailand, P3 performs better than P4, while P2 outperforms P4 for India.

The complex estimator P8 (Ledoit \& Wolf, 2003) fares poorly against the equally weighted portfolio of the sample covariance and singleindex covariance estimator (P2) for India, Indonesia, and the Philippines. P7 (Ledoit \& Wolf, 2004) performs poorly against the equally weighted portfolio of the sample covariance and constant correlation covariance estimator P3 for Indonesia, Pakistan and Thailand. P4 has a lower RMSE 
than P7 and P8 for India, Indonesia and the Philippines. Overall, the results imply that investors have no additional benefit to gain from using a more complex estimator over a simpler, equally weighted portfolio of estimators.

Table 4 gives a risk profile in terms of the standard deviation (SD) of MVPs under the 12 covariance estimators for our sample. We find little variation in their performance under the RMSE and MVP criteria. Again, the sample covariance estimator is a poor estimator, especially for Indonesia, Pakistan and the Philippines. The SDs of the single-index and constant correlation covariance estimators are comparable, although the latter has a lower SD for India, Pakistan and the Philippines.

Table 4: Risk associated with MVP under alternative covariance estimators

\begin{tabular}{lcccccc}
\hline Group & Covariance & India & Indonesia & Pakistan & Philippines & Thailand \\
\hline Conventional & S & 0.0212 & 0.0253 & 0.0256 & 0.0315 & 0.0149 \\
methods & CC & 0.0235 & 0.0244 & 0.0244 & 0.0222 & 0.0165 \\
Factor models & SI & 0.0287 & 0.0241 & 0.0249 & 0.0291 & 0.0163 \\
& PCA & 0.0730 & 0.1677 & 0.1442 & 0.0369 & 0.0971 \\
Portfolio of & P1 & 0.0276 & 0.0225 & 0.0249 & 0.0268 & 0.0176 \\
estimators & P2 & 0.0230 & 0.0234 & 0.0246 & 0.0275 & 0.0157 \\
& P3 & 0.0275 & 0.0241 & 0.0250 & 0.0303 & 0.0156 \\
& P4 & 0.0265 & 0.0235 & 0.0246 & 0.0281 & 0.0159 \\
& P5 & 0.0273 & 0.0228 & 0.0245 & 0.0263 & 0.0168 \\
Shrinkage & P6 & 0.0212 & 0.0251 & 0.0256 & 0.0314 & 0.0149 \\
approaches & P7 & 0.0246 & 0.0248 & 0.0249 & 0.0311 & 0.0152 \\
& P8 & 0.0224 & 0.0236 & 0.0244 & 0.0302 & 0.0165 \\
\hline
\end{tabular}

Source: Authors' calculations.

P2 outperforms the shrinkage estimator P3 for India, Indonesia, Pakistan and the Philippines. For Indonesia, the equally weighted estimators P1, P2, P4 and P5 outperform the complex shrinkage estimators P6, P7 and P8. In Pakistan's case, P1, P2, P4 and P5 fare better than P6 and remain comparable with P7 and P8. In the case of the Philippines, the relatively simple portfolio of estimators $\mathrm{P} 1, \mathrm{P} 2, \mathrm{P} 4$ and $\mathrm{P} 5$ have a lower SD for the MVP than the shrinkage estimators P6, P7 and P8. P3 has an SD of 0.3033, which is almost equal to the SD of P8 (0.0302). P2, P3 and P4 outperform the shrinkage estimator P8 for Thailand.

In India's case, P2 outperforms the shrinkage estimator P8, but P7 has a lower SD than P3, P4 and P5. The equally weighted estimator P2 
performs better than the shrinkage estimator P7 for India, Indonesia, Pakistan and the Philippines. As Table 4 shows, Ledoit and Wolf's (2003, 2004) complex estimator improves relative to the RMSE. On the whole, the equally weighted portfolio group performs better than the shrinkage estimators for this sample, reinforcing the argument that investors will not gain from using a more complex estimator over a portfolio of estimators. Table A1 in the Appendix reports the average mean of the MVP to compare levels of associated risk.

The Sharpe ratio is used to compare the resultant portfolios based on their MVP under alternative covariance estimators (Table 5). This ratio indicates the risk-adjusted return under various inputs to the MVP.

Table 5: Sharpe ratio of resultant MVPs under alternative covariance matrices

\begin{tabular}{llccccc}
\hline Group & Covariance & India & Indonesia & Pakistan & Philippines & Thailand \\
\hline Conventional & S & 0.0381 & 0.1728 & 0.0892 & -0.0100 & 0.2661 \\
methods & CC & 0.1054 & 0.1116 & 0.0971 & 0.1129 & 0.2224 \\
Factor models & SI & 0.0239 & 0.1339 & 0.0682 & 0.0159 & 0.2395 \\
& PCA & 0.1339 & 0.0403 & -0.0323 & 0.2071 & 0.0212 \\
Portfolio of & P1 & 0.0240 & 0.1138 & 0.0666 & 0.0207 & 0.2218 \\
estimators & P2 & 0.0542 & 0.1411 & 0.0926 & 0.0317 & 0.2447 \\
& P3 & 0.0233 & 0.1537 & 0.0792 & 0.0011 & 0.2538 \\
& P4 & 0.0365 & 0.1407 & 0.0852 & 0.0243 & 0.2433 \\
Shrinkage & P5 & 0.0323 & 0.1214 & 0.0755 & 0.0336 & 0.2300 \\
approaches & P7 & 0.0376 & 0.1716 & 0.0889 & -0.0097 & 0.2659 \\
& P8 & 0.0268 & 0.1685 & 0.0713 & -0.0069 & 0.2625 \\
\hline
\end{tabular}

Source: Authors' calculations.

The table shows that the sample covariance performs better than the constant correlation covariance for Indonesia and Thailand. The singleindex covariance outperforms the PCA estimators for Indonesia, Pakistan and Thailand. Among the portfolio of estimators, P2 and P3 outperform the other estimators for Indonesia and Thailand. P8 yields a higher Sharpe ratio than P6 and P7 in the case of India. The evidence is, therefore, mixed: no one estimator consistently outperforms the others, implying that investors gain no additional benefit from using complex estimators. 


\section{Discussion}

Jagannathan and Ma (2003) argue that the equally weighted average of the sample covariance and single-index covariance estimator outperforms the weighted average of the covariance estimator based on optimal shrinkage intensity, as proposed by Ledoit and Wolf (2003). Our findings are consistent with this. Moreover, in line with Liu and Lin (2010), we find that the estimator proposed by Ledoit and Wolf $(2003,2004)$ is better than the equally weighted estimator when gauged by the MVP criterion compared to the RMSE. Jagannathan and Ma (2003) also suggest that constraints in any sense - right or wrong - decrease portfolio risk.

The sample covariance matrix remains a poor estimator based on the RMSE and MVP risk criteria. Given that Ledoit and Wolf's $(2003,2004)$ estimators both depend on the minimization of the quadratic loss function, they should, theoretically, outperform all other weighted estimators. However, consistent with Disatnik and Benninga (2007), we find that the portfolio of weighted estimators based on the optimal shrinkage intensity does not outperform the equally weighted portfolio of estimators for our sample of emerging Asian economies.

Our study reinforces Disatnik and Benninga's (2007) claim that the Ledoit and Wolf $(2003,2004)$ covariance estimator yields a new type of error, which eliminates the benefit of using a weighted portfolio of covariance estimators based on the optimal shrinkage intensity. Further, it offers no additional benefits over using the equally weighted average of covariance estimators in this case.

\section{Conclusion}

This study adopts the implementation approach to portfolio optimization. We compare 12 covariance estimators across four categories conventional methods, factor models, portfolios of estimators and the shrinkage approach - applied to five emerging Asian economies (India, Indonesia, Pakistan, the Philippines and Thailand). The data used is drawn from ten sectors classified under the GICS. We use two different criteria to compare the covariance estimators: the RMSE to establish accuracy and portfolio allocation to gauge their effectiveness.

We find that the sample covariance matrix is a poor estimator in terms of the RMSE and MVP, while the equally weighted average of covariance estimators performs better than the more complex shrinkage 
estimators proposed by Ledoit and Wolf $(2003,2004)$. The covariance estimator also yields a different conclusion under both the RMSE and MVP criteria. In the context of our sample, simpler covariance estimators perform better under the RMSE than more complicated covariance estimators. The opposite holds when applying the MVP criterion.

This implies that, in general, investors gain no advantage in using more complex estimators over a simpler, equally weighted portfolio of estimators in emerging Asian countries. Both investors and portfolio managers should, therefore, consider the portfolio of estimators and factor models better benchmarks than other, more sophisticated estimators.

Subsequent research could take into account the impact of higherorder moments when formulating optimal portfolios. We also recommend that investors develop better comparison criteria for the variancecovariance matrix because the RMSE only considers individual differences in each element of the matrix, while a better gauge would look at its overall structure. Moreover, the MVP is only one portfolio on the efficient frontier in terms of asset allocation. This means that other criteria are needed to compare different covariance estimators for more satisfactory results. 


\section{References}

Bengtsson, C., \& Holst, J. (2002, November). On portfolio selection: Improved covariance matrix estimation for Swedish asset returns. Paper presented at the 31st Meeting of the EURO Working Group for Commodities and Financial Modeling, Cyprus.

Blume, M. E. (1971). On the assessment of risk. The Journal of Finance, 26(1), 1-10.

Chan, L. K., Karceski, J., \& Lakonishok, J. (1999). On portfolio optimization: Forecasting covariances and choosing the risk model. Review of Financial Studies, 12(5), 937-974.

DeMiguel, V., Garlappi, L., \& Uppal, R. (2009). Optimal versus naive diversification: How inefficient is the $1 / \mathrm{N}$ portfolio strategy? Review of Financial Studies, 22(5), 1915-1953.

Disatnik, D. J., \& Benninga, S. (2007). Shrinking the covariance matrix. Journal of Portfolio Management, 33(4), 55-63.

Elton, E. J., \& Gruber, M. J. (1973). Estimating the dependence structure of share prices: Implications for portfolio selection. The Journal of Finance, 28(5), 1203-1232.

Jagannathan, R., \& Ma, T. (2003). Risk reduction in large portfolios: Why imposing the wrong constraints helps. The Journal of Finance, 58(4), $1651-1684$.

Jobson, J. D., \& Korkie, B. (1980). Estimation for Markowitz efficient portfolios. Journal of the American Statistical Association, 75(371), 544-554.

Jorion, P. (1986). Bayes-Stein estimation for portfolio analysis. Journal of Financial and Quantitative Analysis, 21, 279-292.

King, B. F. (1966). Market and industry factors in stock price behavior. The Journal of Business, 39(1, Pt. 2), 139-190.

Kwan, C. C. (2011). An introduction to shrinkage estimation of the covariance matrix: A pedagogic illustration. Spreadsheets in Education, 4(3), art. 6. 
Kyj, L., Ostdiek, B., \& Ensor, K. (2010, January). Covariance estimation in dynamic portfolio optimization: A realized single-factor model. Paper presented at the Annual Meeting of the American Finance Association, Atlanta, GA.

Ledoit, O., \& Wolf, M. (2003). Improved estimation of the covariance matrix of stock returns with an application to portfolio selection. Journal of Empirical Finance, 10(5), 603-621.

Ledoit, O., \& Wolf, M. (2004). Honey, I shrunk the sample covariance matrix. Journal of Portfolio Management, 30(4), 110-119.

Liu, L., \& Lin, H. (2010). Covariance estimation: Do new methods outperform old ones? Journal of Economics and Finance, 34(2), 187-195.

Markowitz, H. (1952). Portfolio selection. The Journal of Finance, 7(1), 77-91.

Markowitz, H. (1999). The early history of portfolio theory: 1600-1960. Financial Analysts Journal, 55(4), 5-16.

Michaud, R. O. (1989). The Markowitz optimization enigma: Is 'optimized' optimal? Financial Analysts Journal, 45(1), 31-42.

Pafka, S., \& Kondor, I. (2004). Estimated correlation matrices and portfolio optimization. Physica A: Statistical Mechanics and its Applications, 343, 623-634.

Sharpe, W. F. (1963). A simplified model for portfolio analysis. Management Science, 9(2), 277-293.

Stein, C. (1956). Inadmissibility of the usual estimator for the mean of a multivariate normal distribution. In J. Neyman (Ed.), Proceedings of the Third Berkeley Symposium on Mathematical and Statistical Probability (pp. 197-206). Berkeley, CA: University of California.

Vasicek, O. A. (1973). A note on using cross-sectional information in Bayesian estimation of security betas. The Journal of Finance, 28(5), 1233-1239.

Wolf, M. (2004). Resampling vs. shrinkage for benchmarked managers (Technical Article No. 4). Zurich: University of Zurich. Retrieved from http://www.econ.uzh.ch/dam/jcr:ffffffff-935a-b0d6-00000000487f72c5/wilmott2007.pdf 
Appendix

\section{Detailed results for MVPs}

This study also uses the risk associated with MVPs as a criterion for comparison. We compute the weights by MVP under the alternative covariance matrix, based on which we record the return on the MVP in an out-of-sample window. Finally, we calculate the mean returns for this series (Table A1).

\section{Table A1: Average means for MVPs}

\begin{tabular}{lcccccc}
\hline Group & Covariance & India & Indonesia & Pakistan & Philippines & Thailand \\
\hline Conventional & S & 0.0008 & 0.0044 & 0.0023 & -0.0003 & 0.0040 \\
methods & CC & 0.0025 & 0.0027 & 0.0024 & 0.0025 & 0.0037 \\
Factor models & SI & 0.0007 & 0.0032 & 0.0017 & 0.0005 & 0.0039 \\
& PCA & 0.0098 & 0.0068 & -0.0047 & 0.0076 & 0.0021 \\
Portfolio of & P1 & 0.0007 & 0.0026 & 0.0017 & 0.0006 & 0.0039 \\
estimators & P2 & 0.0012 & 0.0033 & 0.0023 & 0.0009 & 0.0038 \\
& P3 & 0.0006 & 0.0037 & 0.0020 & 0.0000 & 0.0040 \\
& P4 & 0.0010 & 0.0033 & 0.0021 & 0.0007 & 0.0039 \\
& P5 & 0.0009 & 0.0028 & 0.0019 & 0.0009 & 0.0039 \\
Shrinkage & P6 & 0.0008 & 0.0043 & 0.0023 & -0.0003 & 0.0040 \\
approaches & P7 & 0.0007 & 0.0042 & 0.0018 & -0.0002 & 0.0040 \\
& P8 & 0.0010 & 0.0037 & 0.0023 & 0.0001 & 0.0037 \\
\hline
\end{tabular}

Source: Authors' calculations. 